\title{
Pitfalls in Imaging for Acute Scrotal Pathology
}

\author{
Michele Bertolotto, MD, 'Vito Cantisani, MD, ${ }^{\dagger}$ Massimo Valentino, MD, ${ }^{\ddagger}$ \\ Pietro Pavlica, MD, ${ }^{\S}$ and Lorenzo E. Derchi, MD"
}

\section{Introduction}

$\mathrm{D}$ ifferentiation among the many clinical situations that can present with acute scrotal pain is a significant diagnostic problem in clinical practice. A firm diagnosis is often difficult based on clinical history and physical examinations alone, and imaging is usually requested for this purpose. However, imaging findings alone may not clarify the diagnosis as imaging studies have to be interpreted in close correlation with clinical information and with knowledge of the possible pathologic situations underlying the clinical presentation of the specific patient.

In this article we describe and discuss the most common problems encountered when performing diagnostic studies for acute scrotal symptoms and the solutions for, possibly, avoiding them.

\section{Testicular Torsion}

In patients with testicular torsion ultrasonographic (US) findings vary with time and with the number of twists of the spermatic cord, and both these factors have to be taken into account when performing the study.

Time from beginning of symptoms is important information that has to be obtained from the patient before starting the study.

Early after onset of symptoms, the torsed testis may appear to be of normal volume and echogenicity at US imaging. It takes about 4-6 hours to see it become swollen and hypoechoic, whereas a heterogeneous structural pattern can be observed at about 12 hours (Fig. 1).

*Department of Radiology, University of Trieste, Trieste, Italy.

$\uparrow$ Department of Radiology, Anatomopathology and Oncology, "Sapienza" University of Rome, Rome, Italy.

\#Department of Radiology, Ospedale Sant'Antonio Abate, Tolmezzo, Italy.

§Department of Radiology, Villalba Hospital, Bologna, Italy.

\|Radiology Section, Department of Health Sciences (DISSAL), University of Genoa, Genoa, Italy.

Address reprint requests to Michele Bertolotto, MD, Department of Radiology, University of Trieste, Ospedale di cattinara, Strada di Fiume 447, 34149 Trieste, Italy. E-mail: bertolot@units.it
Time is also a critical factor for the demonstration of hyperemia within paratesticular tissues and the scrotal wall at color Doppler. This finding, in fact, is related to the dilatation of superficial vessels in an attempt to open collaterals to the testis and it takes time, usually a few hours, to become visible. ${ }^{1-3}$

The degree of torsion is the other important factor that affects imaging findings.

In patients with acute painful scrotum, if absence of identifiable intratesticular flow is used as the only diagnostic criterion of torsion at color Doppler ultrasound, an 86\%-94\% sensitivity, nearly 100\% specificity, and 97\% accuracy is obtained. ${ }^{4,5}$ Thus, $6 \%-14 \%$ of patients with torsion have a false negative Doppler evaluation. Most of these patients have a low-degree torsion of the spermatic cord. There is only closure of the veins and lymphatics within the spermatic cord whereas the arteries still remain patent so that arterial flow signals are still visible within the affected testis. ${ }^{6-8}$

Arterial testicular flow signals can be present principally near the hilum, but occasionally also in a more peripheral distribution, with variable waveform characteristics depending on the severity of torsion. The clue is detection of asymmetry or number of color Doppler flow signals visible in the affected and contralateral testes. On the symptomatic side, detection of monophasic waveforms, increased resistance index with decreased diastolic flow velocities, or diastolic flow reversal, are all signs of ischemia caused by swelling and edema, which is occluding the venous flow (Fig. 2).

Many cases of low-degree torsion are recognized only after careful examination of the morphologic characteristics of the spermatic cord. Direct visualization of the twisted portion of the spermatic cord as a funicular mass, or noting an abrupt change in the course, size, and shape of the spermatic cord are helpful in making the diagnosis. Twisting occurs just outside the external inguinal ring, at a varying distance above the testis, or posterior to the testis. Demonstration of the funicular vessels wrapping around the central axis of the twisted spermatic cord, described as the "whirlpool sign," is a highly suggestive sign of low-degree torsion. Moving the probe in craniocaudal direction along the axis of the spermatic cord facilitates the identification of this sign. ${ }^{9}$ 

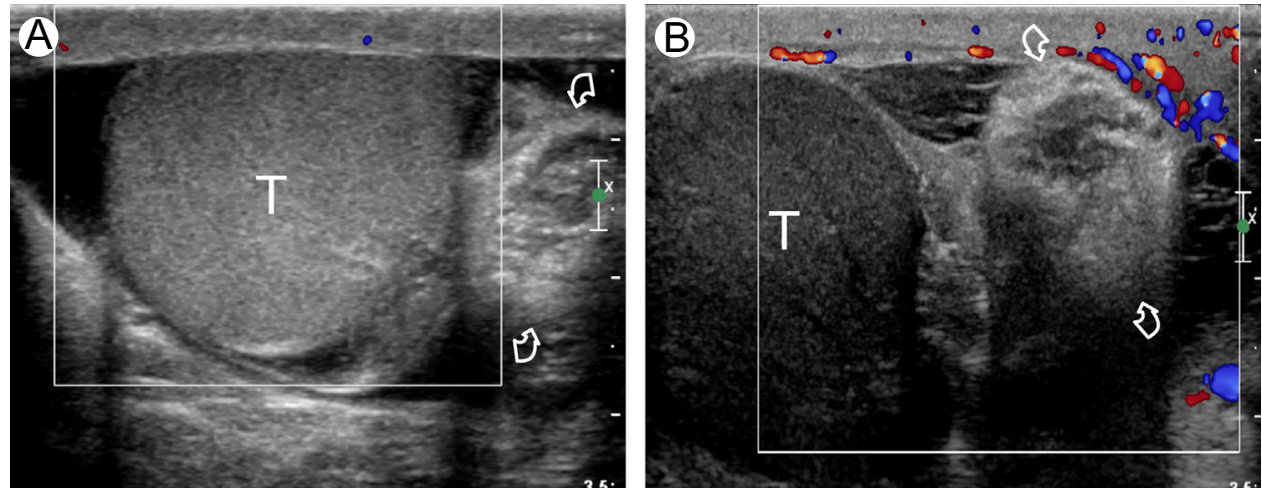

Figure 1 High-degree testicular torsion in two men presenting with right acute scrotal pain for 4 hours (A) and 48 hours (B), respectively. Intratesticular flow signals are absent in both patients. Twisting of the spermatic cord (curved arrows) is appreciable medially to the testis (T). The testis displays normal echogenicity in early torsion, and only few vessels are visible within the scrotal wall (A). At surgery, testis was viable (not shown). In the long-standing torsion the testis ( $\mathrm{T}$ ) is enlarged, markedly hypoechoic, and hyperemia of the paratesticular tissues is recognized (B). At surgery, testis was necrotic. (Color version of figure is available online.)

\section{Postinflammatory Testicular Ischemia}

Epididymo-orchitis is the most common cause of acute scrotal pain in adults and represents at least $75 \%$ of all inflammatory scrotal disease processes. Complications include abscess, testicular ischemia, and pyocele formation. In postinflammatory testicular ischemia, engorgement of the epididymis and testis produces venous outflow obstruction and, eventually, impairment of the arterial blood supply and tissue ischemia. In absence of aggressive medical treatment with antibiotics and anti-inflammatory drugs the disease may progress to segmental or global testicular infarction.

In uncomplicated epididymo-orchitis, the testis and epididymis are hypervascular. In contrast, in patients with postinflammatory ischemia, vascularity of the affected testis is reduced compared to the contralateral one. ${ }^{10}$ Furthermore, intratesticular arteries may show high-resistance signals, and even diastolic flow reversal. ${ }^{11}$ These findings have to raise concern for low-degree testicular torsion; clue to the diagnosis is the clinical history of inflammation, as well as the failure to visualize the twisting of the spermatic cord. Contrast-enhanced ultrasonography (CEUS) provides an excellent evaluation of testicular perfusion in these patients in differentiating necrotic from viable, hypoperfused testes, ${ }^{12-15}$ and showing segmental infarcted areas (Fig. 3). During follow-up it is possible to monitor the efficacy of medical therapy by showing restoration of normal parenchymal vascularization or progression to global infaction or even abscess formation.

\section{Posttraumatic Testicular Torsion}

Testicular torsion is a rare consequence of scrotal trauma that requires immediate surgical intervention. ${ }^{16}$ Clinical manifestations and US appearance are similar to those of nontraumarelated torsion, but the diagnosis is often missed because the pain is incorrectly ascribed to the trauma. Furthermore, the structural and vascular changes within the testis at color Doppler interrogation are misinterpreted as a consequence of edema and hemorrhage of posttraumatic origin.
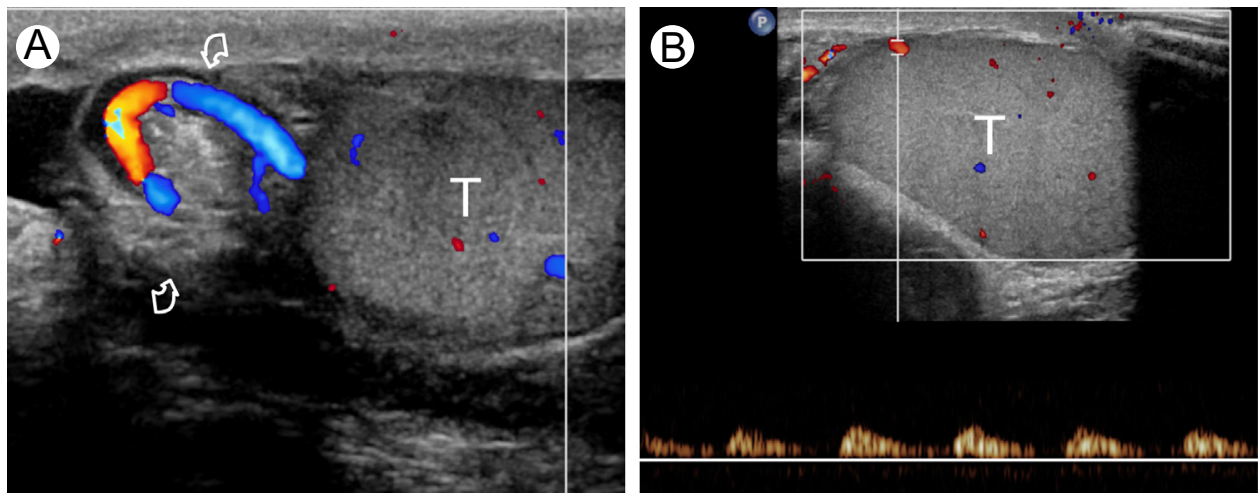

Figure 2 Low-degree testicular torsion. (A) Color Doppler interrogation of the spermatic cord shows vessels with whirlpool course at the level of the twisting of the spermatic cord (curved arrows) consistent with low-degree torsion. (B) Spectral Doppler analysis shows high resistance flows. T: testis. (Color version of figure is available online.) 

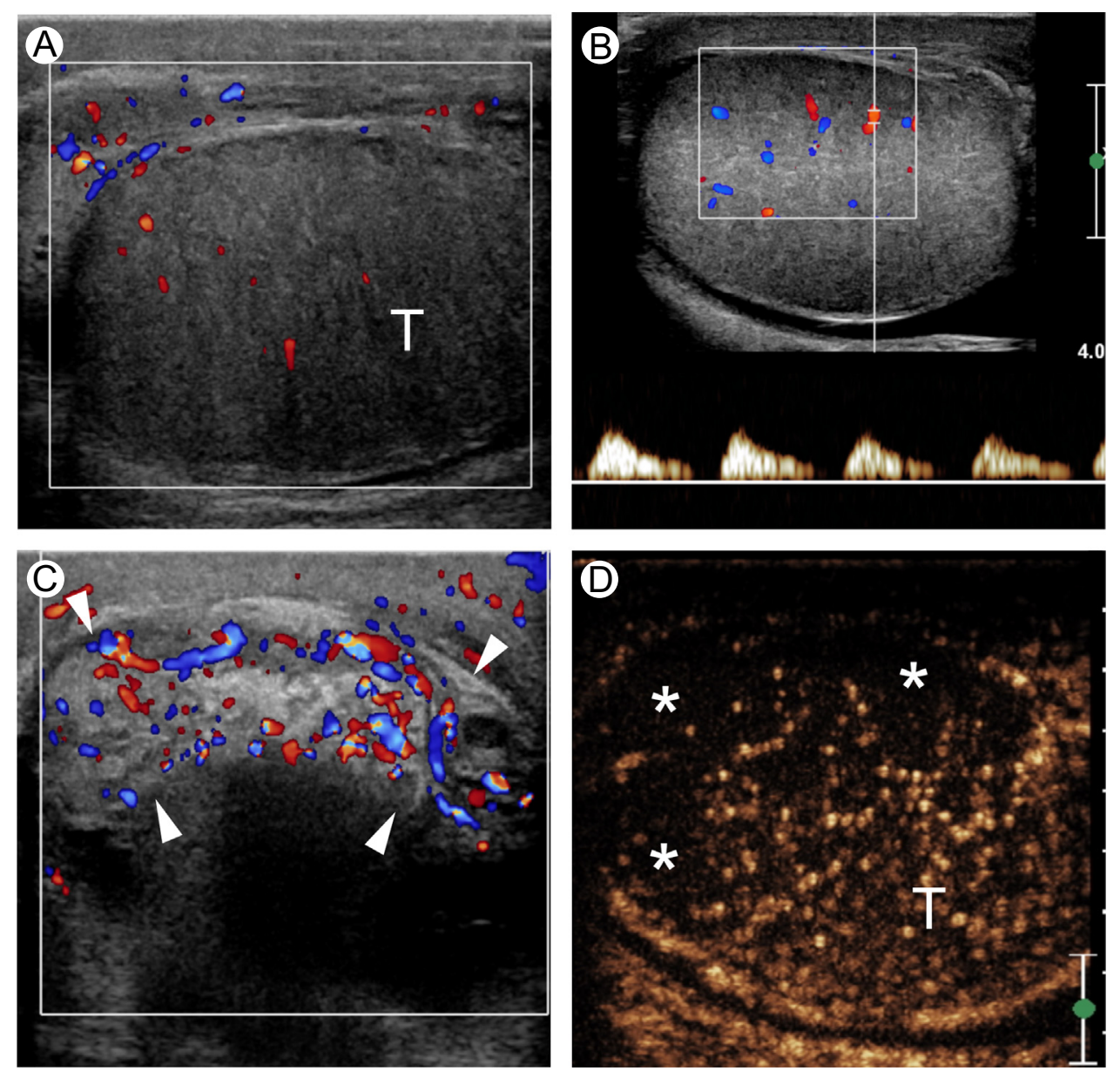

Figure 3 Postinflammatory testicular ischemia in a patients with severe epididymo-orchitis. (A) Color Doppler ultrasonography shows enlarged, hypoechoic, hypovascular testis (T). (B) Spectral Doppler analysis shows high resistance flows, consistent with ischemia. (C) The epididymis (arrowheads) is enlarged, inhomogeneous, and hyperemic. (D) CEUS shows an ischemic area (asterisks) in the upper pole and middle portions of the testis $(\mathrm{T})$. (Color version of figure is available online.)

Although rare, posttraumatic testicular torsion should be considered in patients with trauma patients having severe acute scrotal pain and Doppler findings consistent with ischemia. These features should prompt careful evaluation of the morphology of the spermatic cord. As in nontraumatic torsions, the presence of vascular changes in the absence of alterations at gray-scale ultrasound is indicative of the early stage of pathologic changes and thus, of salvageability of the testis.

\section{Vasculitides}

Testicular vasculitis is a difficult diagnosis. The condition is usually part of a systemic disease, like polyarteritis nodosa (PAN), with damage of medium- and small-size intratesticular arteries resulting in vascular stenoses, thromboses and microaneurysms which are $1-5 \mathrm{~mm}$ in size.

Testicular involvement is usually characterized by acute pain without a history of injury or infection, which may suggest a diagnosis of testicular torsion. ${ }^{17,18}$
It is important to differentiate between PAN and torsion because in PAN, testicular viability is often preserved and patients usually improve on treatment with cyclophosphamide and corticosteroids. Differentiating between these diagnoses by imaging alone in the absence of a history of PAN is difficult, and the diagnosis is often made operatively by the surgeon or unfortunately, on histological examination of the orchiectomy specimen.

Both in PAN and in testicular torsion color Doppler US may fail to show intratesticular vascularization, or can show a marked reduction in arterial blood flow. Echotexture of the involved testis can be heterogeneous, with areas of different echogenicity representing hemorrhage and ischemia. Failure to visualize the twisting of the spermatic cord may help to consider the diagnosis of pseudotesticular torsion in patients with PAN. Churg-Strauss syndrome, a small-vessel necrotizing vasculitis involving the lung, kidneys and other organs, can also present with acute scrotal pain caused by ischemic changes within the testis (Fig. 4).

Acute scrotal pain can also be observed in children with Henoch-Schönlein purpura. ${ }^{19,20}$ At US, testes are usually normal, whereas epididymes are enlarged and heterogeneous. 

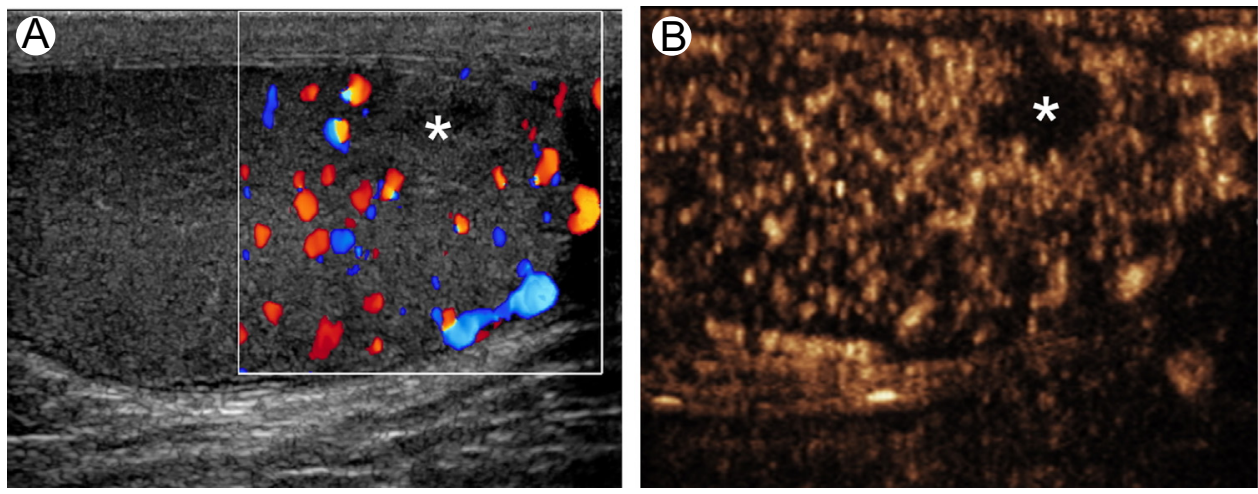

Figure 4 Segmental testicular infarction in a 57-year-old patient with Churg-Strauss syndrome presenting with right acute scrotal pain. (A) Color Doppler interrogation shows a globally hypervascular right testis with an avascular, inhomogeneously hypoechoic area in the lower pole, consistent with infarction. (B) CEUS confirms lack of vascularity of the lesion. (Color version of figure is available online.)

Scrotal skin thickening and reactive hydrocele are usually associated. Color Doppler interrogation shows epididymis hyperemia with normal testicular flow (Fig. 5). Differentiating from epididymitis may be difficult when scrotal involvement presents before the disease is clinically apparent elsewhere

\section{Segmental Testicular Infarction}

Segmental testicular infarction usually presents with acute scrotal pain and may clinically resemble epididymo-orchitis or torsion. If a firm diagnosis of infarction is reached based on imaging features and negative tumor markers, follow-up is advocated. $^{21}$ The condition may present as a solid intratesticular lesion that is hypoechoic or isoechoic to the surrounding normal parenchyma, and is avascular at color Doppler. The key factor that allows differentiation between segmental testicular infarction and tumor is recognizing that

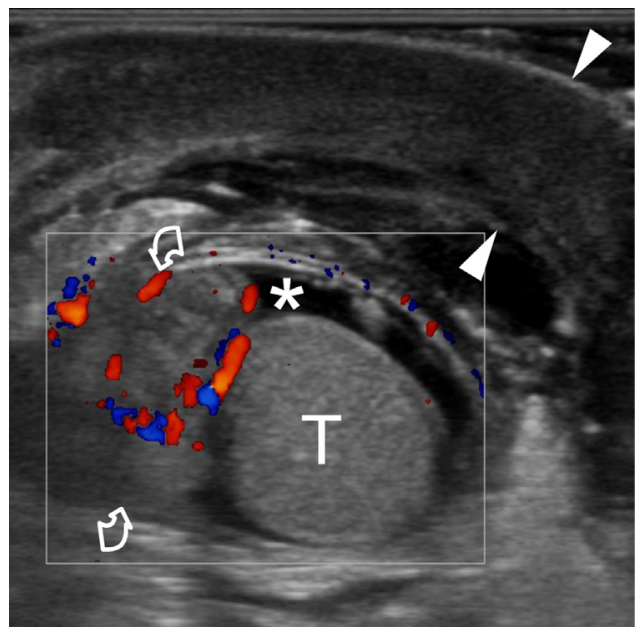

Figure 5 Henoch-Schönlein purpura. Longitudinal color Doppler sonogram of the right hemiscrotum in a 5-year-old boy presenting with acute scrotal pain and scrotal swelling. The epididymis (curved arrows) is enlarged and hyperemic. Thickening of the scrotal wall (arrowheads) and a mild reactive hydrocele (asterisk) is associated. The testis $(\mathrm{T})$ is normal. (Color version of figure is available online.) the lesion is formed by one or more ischemic testicular lobules. Identification of the lobular morphology and the ischemic nature of the abnormality is straightforward when the lesion is wedge shaped. Segmental testicular infarction, however, may be round in shape, and may not be completely avascular at color Doppler interrogation. When intralesional color spots are present, infarction cannot be safely differentiated from hypovascular tumors. Moreover, increased perilesional parenchymal flow signals may be present in segmental infarction, and these are difficult to differentiate from the peripheral vascularization of a tumor, especially in small lesions. It is known that small testicular tumors may appear avascular at color Doppler interrogation. In clinical practice, diagnosis of segmental testicular infarction is often made following orchiectomy for a suspected tumor.

CE magnetic resonance imaging (MRI) is claimed to be the best imaging modality to obtain a definitively diagnosed segmental testicular infarction. The lesion does not enhance following gadolinium administration, and an enhancing perilesional rim can be seen. CEUS improves characterization of acute testicular segmental infarction, depicting the morphological features of this lesion, which are different from those of hypovascular tumors. ${ }^{22}$ Infarction presents as one or more avascular areas separated by normal vessels, consistent with ischemic testicular lobules (Figs. 6 and 7). In contrast to color Doppler US, after microbubble injection, vessels are displayed for a long time, making the differentiation between normal centripetal testicular arteries, arising from the capsular arteries, and tumor neovascularization straightforward.

\section{Focal Testicular Lesions in Patients With Trauma or Infection}

Tumors can occasionally manifest with pain, ${ }^{23}$ or can be found occasionally in patients with trauma. In approximately $10 \%$ of the patients with testicular neoplasms, in fact, symptoms may be misleading and suggest an inflammatory process. The US findings may be unhelpful as well. Differentiating between focal intratesticular lesions such as focal orchitis, focal 

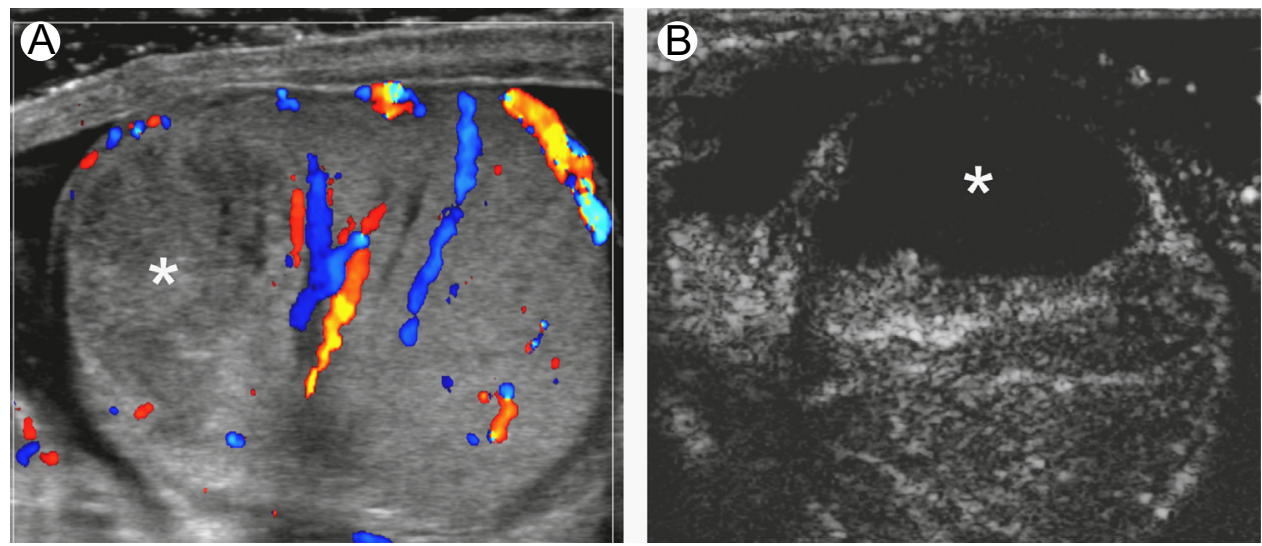

Figure 6 Segmental testicular infarction in a patient presenting with right acute scrotal pain for 3 days. (A) Color Doppler ultrasonography reveals an inhomogenously hypoechoic area (asterisk) in the upper pole of the testis which lacks vascularization at color Doppler interrogation, consistent with segmental infarction. (B) CEUS shows a completely avascular lesion (asterisk). (Color version of figure is available online.)

infarction, tumor, as well as between a testicular abscess and a necrotic tumor may be difficult, especially if the abscess has been already partially treated.

Tumor markers often help in differentiating between testicular tumors and nonneoplastic lesions. Virtually all patients with choriocarcinoma have elevated human chorionic gonadotropin levels. Serum alphafetoprotein levels are elevated in approximately half of the patients with nonseminomatous neoplasms. Leydig and Sertoli cell tumors may produce excessive estrogen or testosterone, resulting in precocious virilization, or feminization. Normal serum tumor markers, however, do not rule out testicular neoplasms.

Follow-up examinations may be helpful in arriving at a diagnosis by showing changes, or lack of changes, within the lesions. The US findings of traumatic and inflammatory changes evolve rapidly and may be recognized at short-term follow-up. Hematomas, in particular, change in shape within a few days, reduce in size and disappear in a relatively short time. Meanwhile, testicular tumors either do not change significantly or increase in size over time.

Although lack of visible vessels within a testicular lesion at color Doppler, in association with a perilesional hypervascular rim, suggests a benign process, the confirmation of the diagnosis should be obtained with CEUS, which can help in differentiating tumors from nonenhancing benign entities, such as abscesses, infarcted areas, and hematomas. On CEUS these lesions do not enhance, but the surrounding parenchyma may show avid enhancement. ${ }^{3,12,15,24}$

\section{Lymphoma vs Inflammation}

Lymphoma and other hematological malignancies of the testis enter in the differential diagnosis of changes related to inflammation. Althought germ-cell tumors usually present as a mass that enlarges, destroys or replaces the normal parenchyma, the hallmark of lymphoma is an infiltrative growth pattern in which tumor cells surround and compress the seminiferous tubules and the normal testicular vessels. At ultrasound, lymphoma presents with single or multiple focal regions of decreased echogenicity of variable size, or as a diffuse enlargement and decreased echogenicity of the entire testis; the normal testicular shape is maintained and there may be
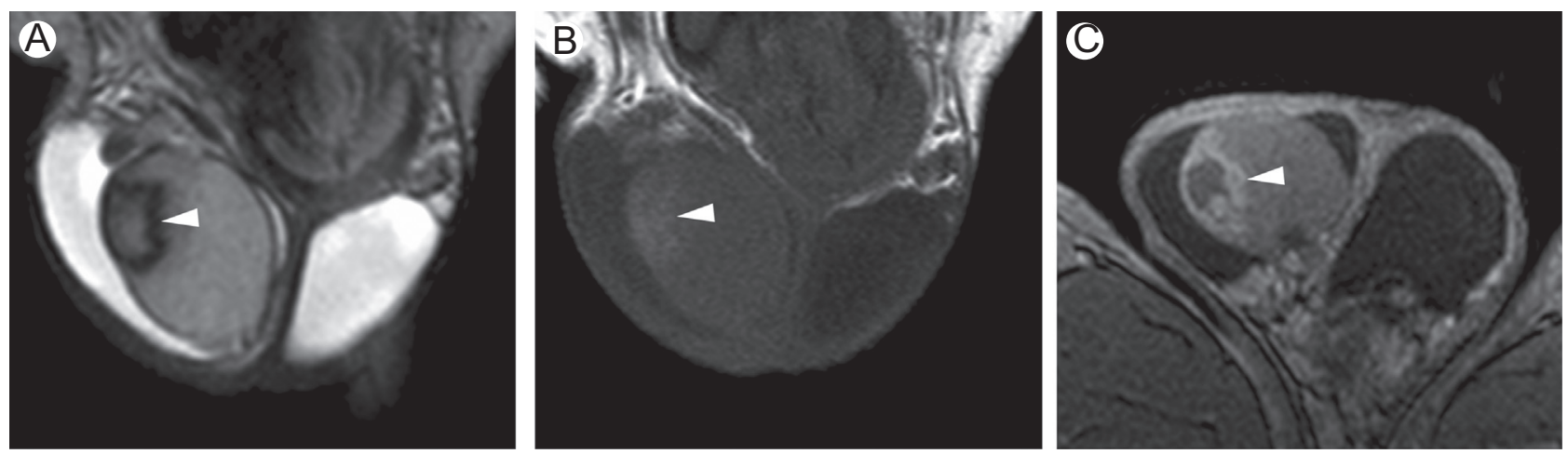

Figure 7 Segmental testicular infarction in a patient presenting with right acute scrotal pain for 12 days. Same case as in Fig. 6, MRI investigation performed 9 days after color Doppler ultrasonography. (A) T2-weighted and (B) T1-weighted coronal images reveal a subacute hemorrhagic area in the right testis (arrowhead). (C) Gadolinium-enhanced axial T1weighted image shows an avascular lesion (arrowhead) with perilesional rim of enhancement. 


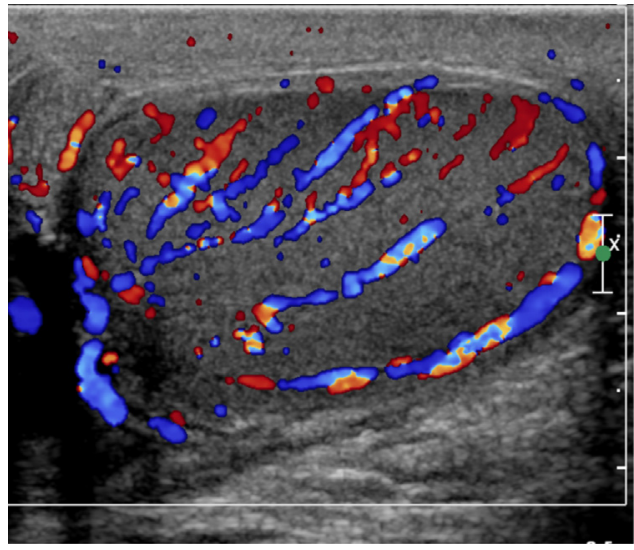

Figure 8 Diffuse large B-cell lymphoma in a 63-year-old patient presenting with progressive enlargement of the left testis for 2 weeks, which was mildly painful. There were no clinical signs of inflammation. Tumor markers were negative. Color Doppler ultrasonography shows diffuse hypervascularization of the left testis and no circumscribed masses. The vascular architecture of the testis is normal. Biopsy revealed diffuse lymphoma infiltration of the parenchyma. (Color version of figure is available online.)

hypervascularization of the entire testis. ${ }^{25}$ Pain may be present, and involvement of the epididymis is common.

In lymphoma the distribution of vessels within the lesion may be relatively normal. ${ }^{25,26}$ Identification of normal testicular vessels with a regular course crossing the tumor tissue is a relatively specific feature characterizing lymphomas and other infiltrative tumors and allowing differentiation from massforming lesions (Fig. 8). In a recently published series of 43 cases of testicular lymphoma, color Doppler US demonstrated normal testicular vessels within the tumor tissue in $72 \%$ of cases. ${ }^{25}$ This feature has been described also in other infiltrative neoplasms like plasmacytoma and leukemia. Importantly, nonneoplastic diseases such as chronic granulomatous orchitis and other inflammatory conditions may present with the same grey-scale and Doppler sonographic features as lymphoma.
Accurate interpretation may therefore be difficult in the absence of clinical signs and symptoms of inflammation or without laboratory evidence of hematologic malignancy. Ultimately, in many of these cases, surgery is necessary; after removal of the testis from the scrotum through an inguinal approach, an intraoperative biopsy with frozen interpretation is obtained, and the decision on how to proceed is based on these results.

\section{Isolated Testicular Hyperemia}

When isolated testicular hyperemia is identified, differentiation has to be made between isolated orchitis and primary hematological malignancies of the testes; the latter often present with diffuse hyperemia and normal vascular architecture. Orchitis usually presents with pain, whereas malignancies do not, but this symptom in not sensitive nor specific enough to exclude malignancy. Bilateral involvement as well as isolated involvement of the head of the epididymis can be seen in both situations.

Most cases of isolated orchitis are due to mumps infection, but subacute or chronic granulomatous orchitis can occasionally present with pain and diffuse hyperemia of the testis not involving the epididymis (Fig. 9). As most patients with mumps orchitis present with epididymal involvement as well, either diffuse or involving the head only, in clinical practice hematological malignancies are the prevailing causes of isolated hyperemia of the testis. The most common hematologic malignancy to affect the testes is diffuse large B-cell lymphoma.

Although the clue for distinguishing between isolated orchitis and hematological malignancies is in the history and clinical findings, this may be difficult or even impossible in some cases. Mumps orchitis typically presents in children or young adults with acute scrotal pain arising some days after swelling and tenderness of the salivary glands (Fig. 10). In many cases, however, involvement of the salivary gland is
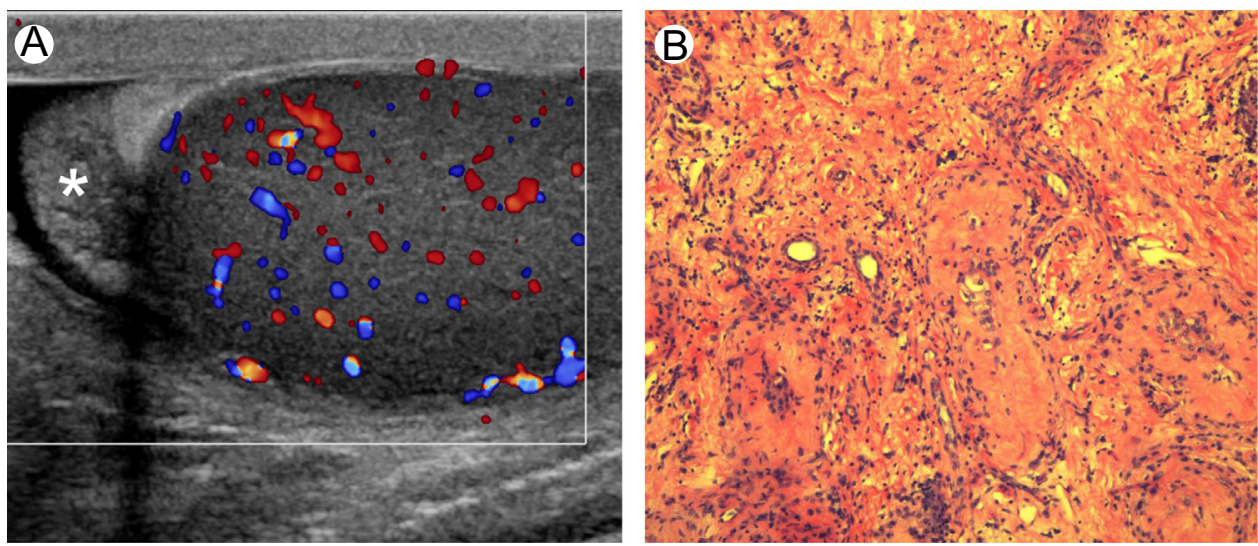

Figure 9 Isolated chronic orchitis in a 65-year-old patient presenting with mildly painful enlargement of the left testis for several weeks and no clinical signs of inflammation. Tumor markers were negative. (A) Color Doppler ultrasonography shows diffuse hypervascularization of the left testis. The epididymis (asterisk) is normal. No changes were observed after 2 weeks of antibiotics. Lymphoma was suspected and biopsy performed. (B) Histologic specimen reveals severe tubular hypothrophy and inflammation. (Color version of figure is available online.) 

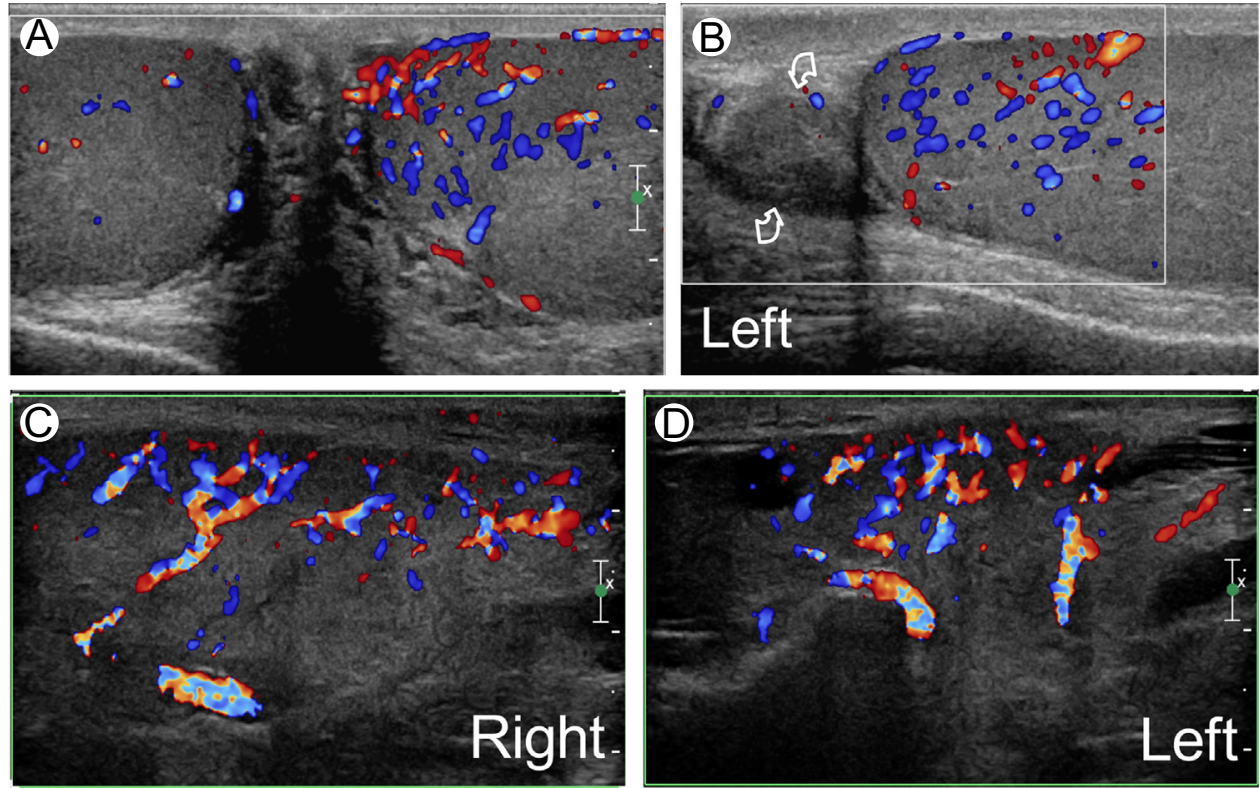

Figure 10 Mumps orchitis. A 25-year-old patient presented with acute left scrotal pain for several hours with no history reported of other disease. (A and B) Color Doppler interrogation shows mild enlargement and hypervascularization of the left testis. The epididymis (curved arrow) is normal. Further questioning revealed that the patient had an US investigation 7 days before for a painless bilateral enlargement of the salivary glands, which were markedly hyperemic (C and D). (Color version of figure is available online.)
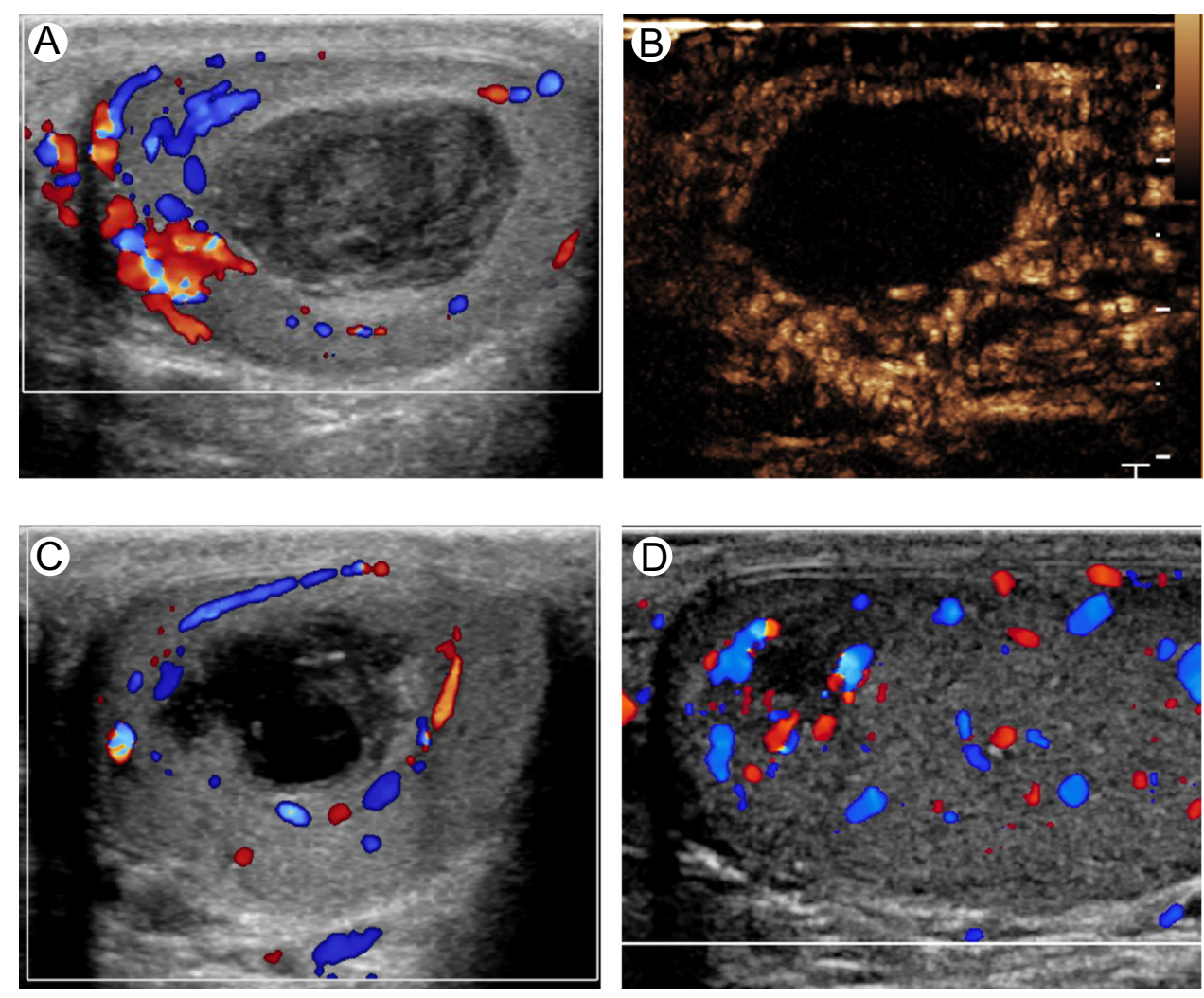

Figure 11 Spontaneous intratesticular hematoma. A 40-year-old patient presented with abrupt onset of severe left testicular pain and swelling. (A) Color Doppler ultrasonography revealed a well defined heterogeneous hypoechoic mass of the left testis without vascularity. (B) CEUS confirms lack of vascularity of the lesion. (C and D) Follow-up Color Doppler ultrasonography performed 2 weeks (C) and 5 months (D) after showed change in lesion echogenicity and progressive reduction in size of the lesion, confirming its benign nature. Hematoma was confirmed with the characteristic appearance of blood at MRI (not shown). (Color version of figure is available online.) 

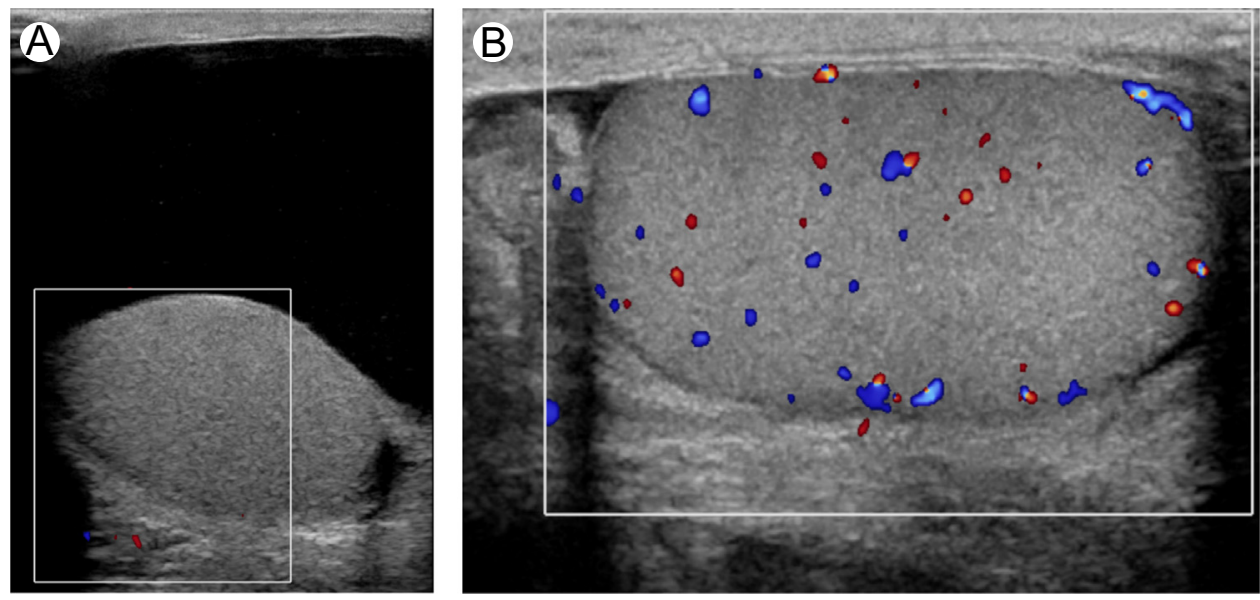

Figure 12 Tension hydrocele. Patient with already known right hydrocele presenting with sudden increase in size of the right hemiscrotum and acute pain. (A) Color Doppler ultrasonography shows avascular right testis. (B) Follow-up investigation obtained after aspiration of the hydrocele shows restoration of the testicular flows. (Color version of figure is available online.)

subclinical, and scrotal involvement is the only clinical manifestation of the disease. Laboratory testing based on detection of the virus-specific immunoglobulin M antibody is essential for confirmation, but is positive in only two-thirds of patients with mumps. ${ }^{27-29}$ Regarding lymphomas, those involving the testis are usually of diffuse large B-cell type. They are more common in men $>60$ years of age, but can occur in younger people. Lymphomas present with progressive swelling of the involved testis, either painful or not, a presentation that can be seen also in chronic and granulomatous orchitis. Ultimately, biopsy is often necessary to establish the diagnosis in the absence of clinical signs and symptoms of inflammation. ${ }^{25}$

\section{Spontaneous Intratesticular Hematoma}

Testicular hematoma is common in the setting of trauma, but can rarely also occur spontaneously. This entity is managed conservatively provided a firm preoperative diagnosis is made. ${ }^{30}$ Patients present with acute scrotal pain without apparent cause and US shows an intratesticular mass suggesting malignancy. However, testicular hematoma is avascular, its appearance changes rapidly over the course of several days, and progressive reduction in size is observed, whereas tumors are vascularized, with a tendency to remain stable in size over time or to enlarge.

The absence of color Doppler signals from an intratesticular solid lesion is not adequate to confidently exclude a hypovascular malignant lesion. Absence of flow must therefore be confirmed with more sensitive imaging techniques such as CEUS or gadolinium-enhanced MRI (Fig. 11). MRI, as an adjunct, has the advantage of showing the signal intensity changes of blood over time, and hemorrhage involving the testicular parenchyma surrounding the hematoma.

\section{Tension Hydrocele}

The hydrostatic pressure of a hydrocele can surpass the pressure of the blood vessels in the testis resulting in ischemia of this organ. ${ }^{31}$

This uncommon condition can be observed in men with a long-standing history of hydrocele that undergoes sudden increase in fluid quantity. Patients present to the emergency department with acute scrotal pain mimicking torsion. On physical examination a large hydrocele is found, that is confirmed at ultrasound. The testis, furthermore, looks compressed from the fluid collection and Doppler interrogation shows avascular testis, high-resistance flows, or diastolic flow reversal. $^{32,33}$ The course of the spermatic cord is normal. In the setting of acute scrotal pain and a large hydrocele, tension hydrocele should be considered to prevent unnecessary surgery. Needle aspiration of the hydrocele results in pain improvement, followed by restoration of low-resistance testicular flows (Fig. 12).

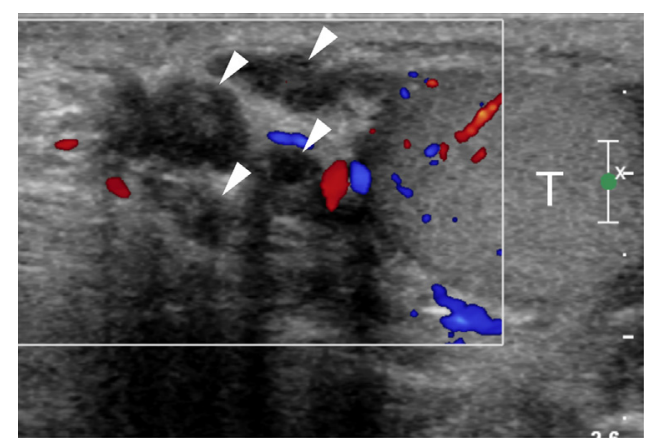

Figure 13 Acute thrombosis of the pampiniform plexus in a patient with severe left testicular pain. A painful lump was palpated above the left testis. Color Doppler ultrasonography shows echogenic material within veins (arrowheads) above the upper pole of the testis ( $\mathrm{T}$ ). (Color version of figure is available online.) 

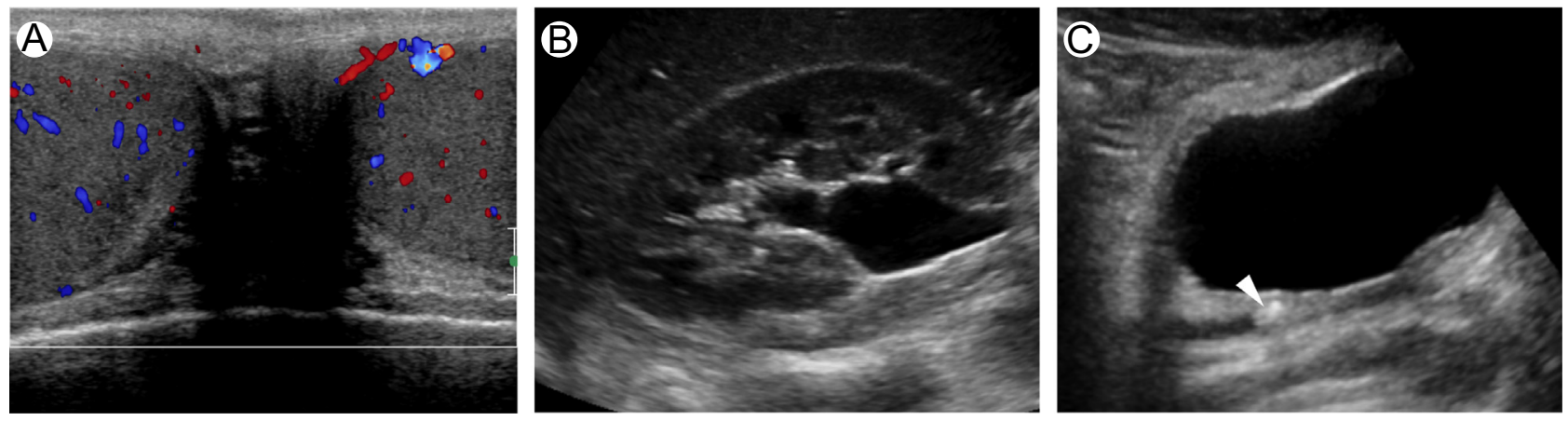

Figure 14 Renal colic in a patient presenting with right acute scrotal pain. (A) Color Doppler interrogation of the scrotum, performed to rule-out testicular torsion, shows normal testes. Extended investigation to the abdomen shows hydronephrosis of the right kidney (B), and a stone (arrowhead) in the pelvic portion of the right ureter (C). (Color version of figure is available online.)

\section{Acute Venous Thrombosis}

Thrombosis of the pampiniform plexus can present clinically as an acutely painful inguino-scrotal mass mimicking an incarcerated hernia. This rare entity can occur spontaneously or be related to exercise in patients with varicocele or with Henoch-Schönlein purpura.

The US and color Doppler interrogation show patent arteries and thrombosed veins, recognized by the presence of echogenic, noncompressible material within the lumen (Fig. 13).

\section{Nonscrotal Causes of Acute Scrotal Pain}

A variety of clinical problems that are not primarily caused by scrotal lesions may cause acute scrotal pain, thus suggesting an acute scrotum. ${ }^{34}$ It is well-known that patients with renal colic from ureterolithiasis may present sometimes with acute scrotal pain and very mild (or even absent) flank pain. These patients are referred for a scrotal US examination because the testis appears clinically to be the organ of concern. In patients presenting with acute scrotal pain in whom all scrotal structures are normal, an US investigation of the kidneys is recommended to rule-out renal colic as a nonscrotal cause for the symptoms (Fig. 14).

There are other extra-scrotal causes of acute scrotal pain. When infected peritoneal fluid or hemoperitoneum develops in a patient with a patent peritoneovaginalis duct, pus or blood can enter the scrotum and cause scrotal enlargement with possible inflammatory symptoms. Retroperitoneal collections caused by pancreatitis or adrenal hemorrhages can dissect inferiorly along the retroperitoneum and involve the superficial layers of the perineum and scrotum, causing enlargement and changes in color of the skin that may suggest an acute scrotal condition. In both cases, scrotal changes can be observed on US that can affect the quantity and quality of fluid within the tunica vaginalis or produce thickening of the layers of the scrotal wall and skin reddening. In both cases, lack of associated changes of the testis and epididymis has to suggest an "unusual" condition and should lead to performance of abdominal studies that can identify the correct diagnosis.

\section{References}

1. Dogra VS, Bhatt S, Rubens DJ: Sonographic evaluation of testicular torsion. Ultrasound Clin 1(1):55-66, 2006

2. Dogra VS, Gottlieb RH, Oka M, et al: Sonography of the scrotum. Radiology 227(1):18-36, 2003

3. Liguori G, Bucci S, Zordani A, et al: Role of US in acute scrotal pain. World J Urol 29(5):639-643, 2011

4. Burks DD, Markey BJ, Burkhard TK, et al: Suspected testicular torsion and ischemia: Evaluation with color Doppler sonography. Radiology 175 (3):815-821, 1990

5. Yagil Y, Naroditsky I, Milhem J, et al: Role of Doppler ultrasonography in the triage of acute scrotum in the emergency department. J Ultrasound Med 29(1):11-21, 2010

6. Lin EP, Bhatt S, Rubens DJ, et al: Testicular torsion: Twists and turns. Semin Ultrasound CT MR 28(4):317-328, 2007

7. Cassar S, Bhatt S, Paltiel HJ, et al: Role of spectral Doppler sonography in the evaluation of partial testicular torsion. J Ultrasound Med 27 (11):1629-1638, 2008

8. Derchi LE, Bertolotto M, Valentino M, et al: The acute scrotum. Ultrasound Clin 8(4):531-544, 2013

9. Vijayaraghavan SB: Sonographic differential diagnosis of acute scrotum: Real-time whirlpool sign, a key sign of torsion. J Ultrasound Med 25 (5):563-574, 2006

10. Yusuf G, Sellars ME, Kooiman GG, et al: Global testicular infarction in the presence of epididymitis: Clinical features, appearances on grayscale, color Doppler, and contrast-enhanced sonography, and histologic correlation. J Ultrasound Med 32(1):175-180, 2013

11. Marks R, McNeil K: Significance of reversal of diastolic blood flow in the evolution of testicular infarction as a complication of epididymo-orchitis. J Radiol Case Rep 3(6):21-25, 2009

12. Cantisani V, Bertolotto M, Weskott HP, et al: Growing indications for CEUS: The kidney, testis, lymph nodes, thyroid, prostate, and small bowel. Eur J Radiol 84(9):1675-1684, 2015

13. Moschouris H, Stamatiou K, Lampropoulou E, et al: Imaging of the acute scrotum: Is there a place for contrast-enhanced ultrasonography? Int Braz J Urol 35(6):692-702, 2009; [discussion 702-5]

14. Piscaglia F, Nolsøe C, Dietrich CF, et al: The EFSUMB Guidelines and Recommendations on the Clinical Practice of Contrast Enhanced Ultrasound (CEUS): Update 2011 on non-hepatic applications. Ultraschall Med 33(1):33-59, 2012

15. Valentino M, Bertolotto M, Derchi LE, et al: Role of contrast enhanced ultrasound in acute scrotal diseases. Eur Radiol 21(9):1831-1840, 2011

16. Papatsoris AG, Mpadra FA, Karamouzis MV: Post-traumatic testicular torsion. Ulus Travma Acil Cerrahi Derg 9(1):70-71, 2003

17. Kolar P, Schneider U, Filimonow S, et al: Polyarteritis nodosa and testicular pain: Ultrasonography reveals vasculitis of the testicular artery. Rheumatology 46(8):1377-1378, 2007 
18. Toepfer NJ, Lountzis NI, Ugoeke JC, et al: Polyarteritis nodosa with bilateral asynchronous testicular necrosis: A case report. Case Rep Urol 2011:465353, 2011

19. Ben-Chaim J, Korat E, Shenfeld O, et al: Acute scrotum caused by Henoch-Schonlein purpura, with immediate response to short-term steroid therapy. J Pediatr Surg 30(10):1509-1510, 1995

20. Coley BD: Sonography of pediatric scrotal swelling. Semin Ultrasound CT MR 28(4):297-306, 2007

21. Bilagi P, Sriprasad S, Clarke JL, et al: Clinical and ultrasound features of segmental testicular infarction: Six-year experience from a single centre. Eur Radiol 17(11):2810-2818, 2007

22. Bertolotto M, Derchi LE, Sidhu PS, et al: Acute segmental testicular infarction at contrast-enhanced ultrasound: Early features and changes during follow-up. Am J Roentgenol 196(4):834-841, 2011

23. Wilson JP, Cooksey G: Testicular pain as the initial presentation of testicular neoplasms. Ann R Coll Surg Engl 86(4):284-288, 2004

24. Bertolotto M, Catalano O: Contrast-enhanced ultrasound: Past, present, and future. Ultrasound Clin 4(3):339-367, 2009

25. Bertolotto M, Derchi LE, Secil M, et al: Grayscale and color Doppler features of testicular lymphoma. J Ultrasound Med 34(6):1139-1145, 2015

26. Bertolotto M, Borsato A, Derchi LE: Lymphoma of the spermatic cord: Sonographic appearance. J Clin Ultrasound 42(8):509-512, 2014
27. Basekim CC, Kizilkaya E, Pekkafali Z, et al: Mumps epididymo-orchitis: Sonography and color Doppler sonographic findings. Abdominal Imaging 25(3):322-325, 2000

28. Tarantino L, Giorgio A, de Stefano G, et al: Echo color Doppler findings in postpubertal mumps epididymo-orchitis. J Ultrasound Med 20 (11):1189-1195, 2001

29. Park SJ, Kim HC, Lim JW, et al: Distribution of epididymal involvement in mumps epididymo-orchitis. J Ultrasound Med 34(6):1083-1089, 2015

30. Gaur S, Bhatt S, Derchi LE, et al: Spontaneous intratesticular hemorrhage: Two case descriptions and brief review of the literature. J Ultrasound Med 30(1):101-104, 2011

31. Wright LA, Gerscovich EO, Corwin MT, et al: Tension hydrocele: Additional cause of ischemia of the testis. J Ultrasound Med 31 (12):2041-2043, 2012

32. Mihmanli I, Kantarci F, Kulaksizoglu H, et al: Testicular size and vascular resistance

before and after hydrocelectomy. Am J Roentgenol 183(5):1379-1385, 2004

33. Turgut AT, Unsal A, Ozden E, et al: Unilateral idiopathic hydrocele has a substantial effect on the ipsilateral testicular geometry and resistivity indices. J Ultrasound Med 25(7):837-843, 2006

34. Napier-Hemy RD, McMahon RF, Payne SR: The acute scrotum: Beware the non-scrotal cause. BJU Int 83(7):878, 1999 